\title{
Propiedades psicométricas de una adaptación de LibQUAL+. Un estudio en bibliotecas de universidades mexicanas
}

\author{
María del Carmen Toledo Sánchez \\ Sonia Elizabeth Maldonado-Radillo \\ Virginia Guadalupe López Torres \\ Universidad Autonoma de Baja California - UABC, México
}

ARTÍCULO / ARTICLE

\section{Resumen}

Objetivo. Validar una escala, de 27 ítems, para diagnosticar la calidad del servicio percibido por los usuarios de la biblioteca de una universidad pública.

Método. Se realizó un estudio en una muestra de 437 usuarios, en el cual se evaluaron las propiedades de una escala adaptada del modelo LibQUAL+. La validez lógica y de contenido fue evaluada por un grupo de expertos (Modelo de Lawshe), mientras que para indagar la estructura subyacente de los ítems se empleó el análisis de componentes principales con rotación Varimax. Resultados. Se identificó la existencia de cuatro variables que explican el 63.038\% de la variación; se descartaron 2 ítems por presentar cargas muy bajas en el análisis factorial. Las pruebas de validez y consistencia interna de la escala son satisfactorias. El instrumento quedó integrado por 25 ítems cuyo Alfa de Cronbach fue 0.929 .

Conclusiones. Se obtuvo un instrumento válido y útil para evaluar la calidad del servicio de la biblioteca en el contexto estudiado.

Palavras-chave

Calidad del servicio ; Bibliotecas universitarias ; Satisfacción de los usuarios ; LibQUAL+ ; México

\section{Pychometric properties from LibQUAL+ adaptation: A study at mexican universities libraries}

Abstract

Objective. The aim of this study is to develop and validate a specific instrument to assess the service quality of the public university library.

Method. The scale was applied to 437 users. A self-administered instrument ( 27 items) was designed to measure the service quality perceived by library users. It was submitted for review by a multidisciplinary group of experts who assessed its logical and content validity (Lawshe's Model). To determine the construct validity, a factor analysis was used with main components and Varimax rotation.

Results. Two items were eliminated from the analysis because they showed a low correlation with the total correlation of the scale. The initial main-components analysis revealed four variables with an explained variation of de $63.038 \%$. The scale has good inner validity and consistency. Consequently, final scale was accepted with 25 items. The global rating of the questionnaire had a Cronbach's alpha of 0.929 .

Conclusions. It is possible to affirm that the referred instrument is a valid and useful approach to evaluate the service quality of library under this specified context.

Keywords

Service quality ; Universities libraries ; Users satisfaction ; LibQUAL+ ; Mexico 


\section{Introducción}

En el transcurso del tiempo ha cambiado la concepción de la calidad en la educación superior, hasta la década del 60 del siglo pasado, se presuponía la calidad basada en la tradición de la institución, en la exclusividad de los profesores, alumnos y recursos materiales; actualmente la situación es distinta porque el fenómeno de la globalización afectó sustancialmente esta concepción, ahora la sociedad está exigiendo una forma diferente de universidad, se exige que el conocimiento impartido sea aplicado a su entorno, que sea pertinente y genere un impacto positivo en éste (Águila, 2005).

Respecto a la gestión de las bibliotecas el tema de la calidad es un fenómeno reciente; Herrera-Viedma, et al. (2008) señalan que la prestación de un servicio óptimo de biblioteca es un factor esencial de calidad del sistema de educación superior europeo. Apoyadas en esta premisa, los gestores de las bibliotecas se han dado a la tarea de elaborar planes estratégicos con la finalidad de obtener la certificación de sus procesos que eleven sus estándares de calidad.

Analizando la literatura publicada sobre evaluación cualitativa, se ha detectado que existen dos tendencias principales en medición de la calidad; una, mediante indicadores de rendimiento (eficacia, eficiencia e impacto) y, la otra, a través de la perspectiva del usuario de los servicios bibliotecarios. Durante las primeras evaluaciones del servicio de las bibliotecas la primera tendencia predominaba; sin embargo hoy en día, la tendencia en la medición es la orientación al usuario final del servicio por tanto la percepción del usuario adquiere gran relevancia (Játiva y Gallo, 2006).

Esto es, en la actualidad la gestión está centrada en el usuario como elemento activo principal de las bibliotecas, por ello, se considera que las bibliotecas son instituciones de servicio, que deben orientarse a satisfacer las necesidades de los usuarios que son la razón de su existencia (Játiva, 2012).

Por lo que se refiere a la evaluación de la calidad de los servicios y gestión de las bibliotecas, actualmente existen diversas normas y estándares internacionales, una de éstas es la norma ISO 11620, la cual en su indicador B.1.1.2, referente a la 'Satisfacción del usuario', enfatiza la importancia de evaluar las percepciones que los usuarios tienen acerca de las horas de atención, las facilidades para el estudio, la disponibilidad de documentos, el servicio de referencia, la capacitación de los usuarios y las actitudes del personal de la biblioteca (ISO, 1998).

Pero, ¿cómo mide la calidad en una biblioteca? Atendiendo a "solo los usuarios juzgan la calidad..." comentado por Zeithaml, Parasuraman, y Berry (1990) entonces una biblioteca será de calidad en la medida que responda a las necesidades de sus usuarios, consecuentemente la medición de la calidad de estos recintos deberá realizarse con base en las opiniones de los usuarios; no obstante, existen otras evaluaciones fundamentadas en criterios objetivos que no son excluyente (Herrera-Viedma, López-Gijón, y Ávila, 2008).

Para medir la calidad del servicio de las bibliotecas se utiliza un instrumento denominado LibQUAL+ desarrollado en el 2000 por un consorcio de 12 bibliotecas americanas junto con la Association of Research Libraries (ARL), se trata de un cuestionario basado en la metodología de evaluación de calidad de servicios denominada ServQUAL (Parasuraman y otros 1985 y 1988) por lo cual también tiene su fundamento en la teoría de la brecha, la cual señala que la valoración de la calidad por parte del usuario se da en tres niveles: las expectativas mínimas (nivel mínimo), las expectativas ideales (nivel deseado) y la realidad (nivel percibido). Este cuestionario destaca por su alto nivel de validez y confiabilidad (Huamán, Alfaro y Vílchez, 2008).

Este estudio pretende profundizar en la problemática de la medición de la calidad del servicio prestado por las bibliotecas de una institución de educación superior. La localización elegida es una universidad pública estatal ubicada en el estado de Baja California de la República Mexicana, la cual es considerada como la más importante en el estado en función del tamaño de su población estudiantil. Actualmente la institución tiene presencia en los cinco municipios del estado, con tres campus ubicados en Ensenada, Mexicali y Tijuana.

El objetivo de esta investigación consiste en verificar las propiedades psicométricas de la adaptacion realizada a LibQUAL+, instrumento para la evaluación de la calidad del servicio bibliotecario en una universidad pública. 
Se pretende contar con un instrumento para evaluar con alto grado de certidumbre qué nivel de calidad tiene el servicio bibliotecario visto desde la perspectiva de sus usuarios cuya aplicación e interpretación sea fácil y sencilla. Esta evaluación es importante porque permitirá analizar comportamientos, demandas y usos, identificar las formas para satisfacer las necesidades, delimitar las condiciones que deben cumplir los servicios ofrecidos y diseñar la programación de formación de usuarios. Pero, ¿para qué evaluar?, además de lo ya mencionado anteriormente, la finalidad principal debe ser la mejora de los servicios en función de la opinión y las necesidades de los usuarios. Los beneficios son claros.

Para la realización de esta investigación se toma como base el cuestionario de LibQUAL+ ampliamente utilizado para determinar la calidad de los servicios bibliotecarios, el cual se adapta al contexto estudiado. El presente trabajo se limita conceptualmente a la calidad del servicio y espacialmente a las bibliotecas de una universidad pública. A fin de lograr el objetivo planteado, el segundo apartado, se refiere al método de investigación que se define como descriptivo e incluye el perfil demográfico de los participantes que contextualizan los hallazgos; en el siguiente apartado de resultados se expone el análisis de la información recabada que se presenta en tablas y; finalmente, se plantean las conclusiones y limitaciones del estudio.

\section{Metodología}

Este trabajo es un estudio descriptivo con diseño no experimental cuyo ámbito de estudio corresponde a una universidad pública (multicampus) del estado de Baja California perteneciente a la región noroeste de la República Mexicana. El universo de estudio se integra por estudiantes de uno de los campus (Ensenada), con bibliotecas distribuidas en tres unidades: Central, Valle Dorado y San Quintín; la selección de los estudiantes se realizó a través de un muestreo por juicio, el cual se lleva a cabo a partir de criterios conceptuales definidos por el investigador (Mejía, 2000).

Para la recolección de los datos se adaptó el cuestionario LibQUAL+ atendiendo a las recomendaciones de Carretero-Dios y Pérez (2005); la adaptación consistió en la adecuación de la redacción de algunos ítems y la inclusión de otros que se consideraron importantes para el contexto. Posteriormente para lograr el objetivo del estudio se trabajó bajo el esquema: Fase I. Evaluación cualitativa de los ítems; Fase II. Análisis estadístico de los ítems; Fase III. Estudio de la dimensionalidad del instrumento y Fase IV. Estimación de la fiabilidad. El instrumento resultante quedo integrado por 25 ítems agrupados en cuatro variables fundamentales cuya técnica de escalamiento es tipo Likert de cinco categorías de respuesta, en el cual las anclas son $1=$ nunca y $5=$ muy frecuentemente.

En el tratamiento estadístico de la información se utilizó el paquete Statistical Package for the Social Siences (SPSS) ® para obtener frecuencias, estadísticos descriptivos, análisis de componentes principales y Alfa de Cronbach. Para determinar la calidad del servicio de la biblioteca se empleó el baremo de intensidad mostrado en la Tabla 2; este baremo se construyó en función del número de ítems medidos en escala ordinal, en donde cada pregunta tiene cinco alternativas (1 al 5) y considerando que solo existe una alternativa de respuesta para cada ítem.

Tabla 2: Baremo de Intensidad para la determinación la Calidad del Servicio

\begin{tabular}{ccc|c}
\hline \multicolumn{2}{|c|}{ Rango } & Nivel \\
\hline 1 & $\longrightarrow$ & Mala \\
\hline 81 & $\longrightarrow$ & 103 & Regular \\
\hline 104 & $\rightarrow$ & 125 & Buena \\
\hline
\end{tabular}

Los intervalos de puntuaciones indicados en este baremo se calcularon considerando la ecuación $0.75 \sigma \pm \mu$. Fuente: Elaboración propia 
Para obtener la evidencia de que la definición semántica está bien representada en los ítems formulados se llevo a cabo la determinación del índice cuantitativo de la validez de contenido del instrumento a través del Modelo de Lawshe modificado por Tristán, en este modelo la Razón de Validez de Contenido (CVR') debe ser igual o superior a 0.5823. El análisis estadístico de los ítems incluyó la determinación del índice de homogeneidad.

La dimensionalidad del instrumento se evalúo a través de un procedimiento exploratorio de análisis factorial que permite determinar el grado en el que los ítems y los componentes del instrumento conforman el constructo que se requiere medir. La fiabilidad se determinó mediante el cálculo del coeficiente de consistencia interna: Alfa de Cronbach (Carretero-Dios y Pérez, 2005).

\section{Resultados}

\subsection{Datos demográficos}

Las características demográficas de la muestra, en cuanto a la procedencia del usuario, estuvo marcada por un predominio de usuarios de la Biblioteca Central con un 53.5\% en tanto que la participación de los usuarios de la Biblioteca de San Quintín fue mínima, pues apenas alcanzó un 2.3\%. Respecto al tipo de alumno, destacan los alumnos de licenciatura de la etapa básica y disciplinaria los cuales cubren 36.9\% y 36\% de la participación, respectivamente; mientras que el personal administrativo alcanza el nivel más bajo $(0.9 \%)$.

\subsection{Análisis de los ítems}

En el análisis estadístico de los ítems se calculó la discriminación de los ítems a través del coeficiente de correlación corregido entre la puntuación en el ítem y la total obtenida en la dimensión a que éste pertenezca (Índice de homogeneidad), la cual se representa en la Tabla 3.

Las correlaciones de cada ítem con todos los demás (correlación ítem total corregida) oscilan entre un mínimo de .381 (i27) y un máximo de .673 (i21). Se observa que aun cuando se encuentran correlaciones bajas, la eliminación del ítem no modifica sustancialmente el Alfa de Cronbach.

\subsection{Determinación de la validez de contenido}

La validez de contenido se realizó a través del modelo de Lawshe (1975), en éste, participaron 6 expertos a los cuales se les pidió que evaluaran cada ítem de acuerdo a su relevancia definida como la importancia del ítem para la determinación de la calidad del servicio que ofrece la biblioteca, atendiendo a la escala: a) esencial, b) útil pero no esencial, y c) no esencial.

Posteriormente con base en el acuerdo de los expertos, se determinó la razón de validez de contenido (CVR y CVR') a partir las ecuaciones 1 y 2, teniendo en cuenta el criterio modificado de Lawshe (Tristán, 2008), los ítems aceptables son aquellos cuya CVR' sea mayor a 0.58 (Heredia, Sánchez y Vargas, 2012). Los resultados se muestran en la Tabla 4. 
Tabla 3: Análisis de los Ítems

\begin{tabular}{|c|c|c|c|}
\hline Ítem & $\begin{array}{c}\text { Correlación } \\
\text { elemento-total } \\
\text { corregida } \\
\end{array}$ & $\begin{array}{l}\text { Correlación } \\
\text { múltiple al } \\
\text { cuadrado }\end{array}$ & $\begin{array}{l}\text { Alfa de Cronbach si se } \\
\text { elimina el elemento }\end{array}$ \\
\hline 1 & .605 & .666 & .926 \\
\hline 2 & .618 & .634 & .926 \\
\hline 3 & .620 & .716 & .926 \\
\hline 4 & .602 & .717 & .926 \\
\hline 5 & .592 & .575 & .926 \\
\hline 6 & .642 & .709 & .926 \\
\hline 7 & .642 & .654 & .926 \\
\hline 8 & .439 & .422 & .928 \\
\hline 9 & .468 & .405 & .928 \\
\hline 10 & .505 & .510 & .928 \\
\hline 11 & .524 & .489 & .927 \\
\hline 12 & .518 & .515 & .927 \\
\hline 13 & .614 & .489 & .926 \\
\hline 14 & .454 & .281 & .928 \\
\hline 15 & .459 & .380 & .928 \\
\hline 16 & .474 & .449 & .928 \\
\hline 17 & .484 & .459 & .928 \\
\hline 18 & .545 & .411 & .927 \\
\hline 19 & .572 & .429 & .927 \\
\hline 20 & .670 & .669 & .925 \\
\hline 21 & .673 & .660 & .925 \\
\hline 22 & .591 & .587 & .926 \\
\hline 23 & .640 & .674 & .926 \\
\hline 24 & .489 & .407 & .928 \\
\hline 25 & .470 & .407 & .928 \\
\hline 26 & .652 & .657 & .925 \\
\hline 27 & .381 & .200 & .929 \\
\hline
\end{tabular}

En esta tabla se muestran los Índices de homogeneidad de los ítems que componen el instrumento, aun cuando se encuentran correlaciones bajas la eliminación del ítem no modifica el valor del índice de consistencia interna. 
Ecuación 1

$$
\begin{aligned}
C V R=\frac{\mathrm{n}_{\mathrm{e}}-\mathrm{N} / 2}{\mathrm{~N} / 2} & \mathrm{~N}=\begin{array}{l}
\text { donde: } \mathrm{n}_{\mathrm{e}}=\begin{array}{l}
\text { núm. de expertos que tiene } \\
\text { acuerdo en la categoría } \\
\text { esencial }
\end{array} \\
\text { núm. total de expertos }
\end{array}
\end{aligned}
$$

Ecuación utilizada para el cálculo de la razón de validez de contenido para cada ítem

Ecuación 2
$C V R^{\prime}=\frac{\mathrm{CVR}+1}{2}$
donde: $\mathrm{CVR}_{=}$
La razón de validez de contenido para cada ítem

Ecuación utilizada para el cálculo de la razón de validez de contenido para cada ítem (Modelo Lawshe modificado)

Ecuación 3

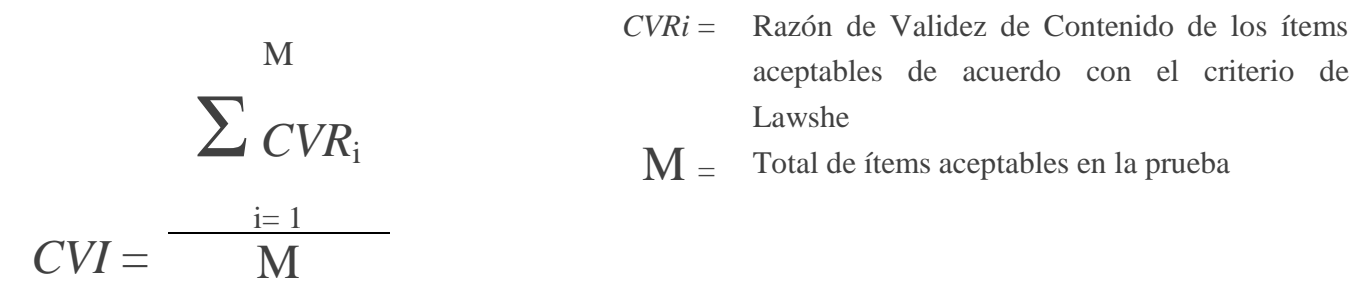

Ecuación empleada para el cálculo de validez global del instrumento

Los valores de la razón de validez de contenido de los ítems demostraron, en su gran mayoría, la validez de contenido es elevada, incluso se encontró que los ítems 1, 3, 4, 6, 12, 16, 17, 21, 25 y 26 fueron valorados con el puntaje máximo de validez del cuestionario (1.00) mientras que solo dos ítems (14 y 27) calificaron por debajo del valor aceptable (Tabla 4).

El valor de la validez global (CVI) del instrumento con el modelo Lawshe calculado con Ecuación 3 fue de 0.81 (ó $81 \%$ ) superior a 0.58 por lo que el instrumento es aceptable en su conjunto (Tristán, 2008).

\subsection{Determinación de la validez de constructo}

Para determinar la dimensionalidad del instrumento se realizó un análisis factorial exploratorio (AFE) de primer grado, utilizando el método de análisis de componentes principales con rotación tipo Varimax verificando previamente que los datos fueron adecuados para este tipo de análisis. Los resultados de la prueba KMO es 0.933 y la prueba de esfericidad de Barlett $\left(C^{2}=6312.655 ; p=.000\right.$ para $\left.300 \mathrm{gl}\right)$ indican que los datos son adecuados para el uso del modelo de análisis factorial.

La revisión de la solución factorial indica que 63.038 \% de la variabilidad total de los ítems es explicada por los cuatro primeros factores; el primer componente (factor) es el que tiene la varianza más alta y por lo tanto la mayor capacidad explicatoria de los datos, en este caso alcanza el $20.95 \%$ del total mucho mayor que el segundo factor $(17.66 \%)$. 
Tabla 4: Razón de Validez de Contenido (Modelo Lawshe)

\begin{tabular}{|c|c|c|c|c|c|c|}
\hline Ítem & Esencial & $\begin{array}{l}\text { Útil/No } \\
\text { esencial }\end{array}$ & $\begin{array}{c}\text { No } \\
\text { importante }\end{array}$ & & CVR & CVR' \\
\hline 1 & 6 & 0 & 0 & & 1.00 & 1.00 \\
\hline 2 & 5 & 0 & 1 & & 0.67 & 0.83 \\
\hline 3 & 6 & 0 & 0 & & 1.00 & 1.00 \\
\hline 4 & 6 & 0 & 0 & & 1.00 & 1.00 \\
\hline 5 & 4 & 2 & 0 & & 0.33 & 0.67 \\
\hline 6 & 6 & 0 & 0 & & 1.00 & 1.00 \\
\hline 7 & 5 & 1 & 0 & & 0.67 & 0.83 \\
\hline 8 & 4 & 2 & 0 & & 0.33 & 0.67 \\
\hline 9 & 5 & 1 & 0 & & 0.67 & 0.83 \\
\hline 10 & 5 & 1 & 0 & & 0.67 & 0.83 \\
\hline 11 & 5 & 1 & 0 & & 0.67 & 0.83 \\
\hline 12 & 6 & 0 & 0 & & 1.00 & 1.00 \\
\hline 13 & 5 & 1 & 0 & & 0.67 & 0.83 \\
\hline 14 & 3 & 1 & 2 & & 0.00 & 0.50 \\
\hline 15 & 4 & 2 & 0 & & 0.33 & 0.67 \\
\hline 16 & 6 & 0 & 0 & & 1.00 & 1.00 \\
\hline 17 & 6 & 0 & 0 & & 1.00 & 1.00 \\
\hline 18 & 5 & 1 & 0 & & 0.67 & 0.83 \\
\hline 19 & 5 & 1 & 0 & & 0.67 & 0.83 \\
\hline 20 & 5 & 1 & 0 & & 0.67 & 0.83 \\
\hline 21 & 6 & 0 & 0 & & 1.00 & 1.00 \\
\hline 22 & 4 & 1 & 1 & & 0.33 & 0.67 \\
\hline 23 & 5 & 0 & 1 & & 0.67 & 0.83 \\
\hline 24 & 4 & 2 & 0 & & 0.33 & 0.67 \\
\hline 25 & 6 & 0 & 0 & & 1.00 & 1.00 \\
\hline 26 & 6 & 0 & 0 & & 1.00 & 1.00 \\
\hline 27 & 3 & 1 & 2 & & 0.00 & 0.50 \\
\hline SUMA & 34 & 4 & 4 & $S=$ & 4.33 & 5.67 \\
\hline \multirow{2}{*}{\multicolumn{4}{|c|}{$\begin{array}{r}\text { Todos: } \\
\text { Ítems } \\
\text { Aceptables }\end{array}$}} & $\mathrm{CVI}=$ & 0.62 & 0.81 \\
\hline & & & & $\mathrm{CVI}=$ & 1 & 0.78 \\
\hline
\end{tabular}

La validez global del instrumento(CVI), atendiendo al criterio de 6 revisores, es del $81 \%$ por lo que el instrumento es aceptable

En la Tabla 5 se dan a conocer, los resultados del análisis factorial utilizando el método de rotación Varimax el cual tiende a forzar la diferenciación y permite la obtención de una estructura subyacente más clara (Morales, 2011). En el primer factor se explica el $20.95 \%$ de la variabilidad de los datos y se observa que todos los ítems (1, 2, 3, 4, 5,6 y 7) hacen referencia a las actitudes del personal con los usuarios por tanto está relacionada con su compromiso. En el segundo factor se detectan los ítems relacionados con las características físicas de la biblioteca como un lugar para realizar actividades de estudio, aquí hay cargas factoriales altas en los ítems 20, 21, 22, 23, 24,25 y 26 . El tercer componente destaca la percepción de los usuarios respecto a los recursos que proporciona la biblioteca encontrando que poseen en el factor cargas altas los ítems 8, 9, 10,11, 12 y 13 . Finalmente se encontró un cuarto factor en el cual los ítems de mayor ponderación son 15, 16, 17, 18 y 19 que tienen que ver con las tecnologías de la información y comunicación aplicadas al servicio. 


\section{Tabla 5: Matriz de los componentes rotados}

\begin{tabular}{|c|c|c|c|c|}
\hline \multirow[t]{2}{*}{ Ítems } & \multicolumn{4}{|c|}{ Factores } \\
\hline & 1 & 2 & 3 & 4 \\
\hline 4. El personal muestra disposición para ayudarlo & .859 & & & \\
\hline 3. El personal es amable en la atención hacia usted & .833 & & & \\
\hline 6. El personal comprende sus necesidades & .831 & & & \\
\hline 1. El personal de la biblioteca le inspira confianza & .817 & & & \\
\hline 2. El personal le ofrece atención personalizada & .795 & & & \\
\hline 7. El personal brinda servicios con prontitud & .786 & & & \\
\hline 5. Considera que el personal posee amplios conocimientos para responder a sus preguntas & .738 & & & \\
\hline 26. La biblioteca es un lugar ideal para estudiar y aprender & & .792 & & \\
\hline 23.La biblioteca otorga un ambiente favorable para el estudio y trabajo en grupo & & .789 & & \\
\hline 20. La infraestructura de la biblioteca ayuda al estudio y el aprendizaje & & .752 & & \\
\hline 22. La biblioteca ofrece un ambiente tranquilo para el estudio individual & & .747 & & \\
\hline 21. La biblioteca cuenta con espacios cómodos & & .746 & & \\
\hline 24. La biblioteca cuenta con una adecuada iluminación & & .665 & & \\
\hline 25. La biblioteca cuenta con una adecuada ventilación & & .661 & & \\
\hline 8. Los libros impresos existentes en la biblioteca cubren las necesidades de información que necesito & & & .740 & \\
\hline 9. La cantidad de copias de los libros impresos son suficientes para mis necesidades de información & & & .732 & \\
\hline 10. Los libros electrónicos existentes en la biblioteca cubren las necesidades de información que necesito & & & .669 & \\
\hline 11. Las revistas de la biblioteca cubren las necesidades de información & & & 647 & \\
\hline 12. Las bases de datos electrónicas de la biblioteca cubren las necesidades de información que necesito & & & .616 & \\
\hline $\begin{array}{l}\text { 13. La colección de recursos informativos (libros impresos, libros electrónicos, etc..) disponibles en la } \\
\text { biblioteca corresponden a ediciones actualizadas? }\end{array}$ & & & .558 & \\
\hline $\begin{array}{l}\text { 16. Puedo accesar a recursos electrónicos (bases de datos, libros electrónicos) desde mi hogar o lugar de } \\
\text { trabajo }\end{array}$ & & & & .763 \\
\hline 17. La biblioteca me permite el acceso a textos completos de revistas usando las bases de datos electrónicas & & & & .711 \\
\hline $\begin{array}{l}\text { 15. El sitio WEB de la biblioteca http://biblioteca.uabc.mx (herramienta para recuperación de información } \\
\text { como catalogo cimarrón, bases de datos, libros electrónicos) es fácil de usar y permite encontrar por mi } \\
\text { mismo lo que busco. }\end{array}$ & & & & .655 \\
\hline $\begin{array}{l}\text { 19. Los cursos de orientación para usuarios, sobre el uso de servicios y recursos de información de la } \\
\text { biblioteca, me permiten utilizar los servicios de biblioteca de forma más eficiente }\end{array}$ & & & & .589 \\
\hline $\begin{array}{l}\text { 18.La biblioteca cuenta con computadoras modernas que me permiten el acceso fácil a la información que } \\
\text { necesito }\end{array}$ & & & & .504 \\
\hline
\end{tabular}

Método de extracción: Análisis de los componentes principales

Método de rotación: Varimax con Kaiser.

Con base en el análisis de la distribución y las cargas factoriales de los ítems el instrumento quedó conformado por cuatro variables: Compromiso de personal, Biblioteca como espacio, Servicios de información proporcionados y Tecnologías de la información y comunicación aplicadas al servicio (Anexo). 


\subsection{Análisis de la Fiabilidad}

La fiabilidad del instrumento se evaluó la consistencia interna mediante el cálculo del alfa de Cronbach que se utiliza para evaluar la homogeneidad de los distintos ítems de una misma variable y del cuestionario global, encontrando que la fiabilidad global fue de 0.928 .

También fue analizada la fiabilidad de cada una de las subescalas (Tabla 6) del instrumento encontrando que la variable compromiso personal presenta una fiabilidad más alta $(\alpha=0.934)$ respecto a las otras variables; mientras que la variable TIC aplicadas al servicio obtiene el índice de consistencia interna mas bajo $\alpha=0.778$.

\section{Tabla 6: Consistencia interna de la escala}

\begin{tabular}{l|c|c}
\hline $\begin{array}{c}\text { Variables de la Calidad del } \\
\text { Servicio }\end{array}$ & $\begin{array}{c}\text { Alfa de Cronbach } \\
\text { (unidad de análisis N=437) }\end{array}$ & $\begin{array}{c}\text { Número de } \\
\text { Ítem }\end{array}$ \\
\hline Compromiso de personal & .934 & 7 \\
\hline Biblioteca como espacio & .899 & 7 \\
\hline Servicio de información proporcionados & .836 & 6 \\
\hline TIC aplicadas al servicio TOTAL: & .778 & 5 \\
\hline
\end{tabular}

Aquí se describen los índices de confiabilidad de las variables compromiso personal, biblioteca como espacio, servicio de información proporcionado y TIC aplicada al servicio así como el número de ítems que conforman cada una de las variables. Fuente: Elaboración propia, cálculo efectuado a través del SPSS 19

\subsection{Valoración de la calidad de los servicios}

El nivel de calidad del servicio de la biblioteca se determinó comparando el puntaje obtenido contra el baremo de intensidad (Tabla 2) construido para tal fin. El análisis reveló que el promedio de puntaje en esta evaluación fue de 91.93 (Tabla 7) un nivel regular lo cual pone de manifiesto que existen áreas de oportunidad que deben ser aprovechadas para la elaboración de un plan de mejora continua.

Tabla 7: Estadísticos Descriptivos

\begin{tabular}{l|c|c|c|c|c|c|c}
\hline & $\mathbf{N}$ & $\begin{array}{c}\text { Media } \\
(\mu)\end{array}$ & $\begin{array}{c}\text { Rango } \\
\text { Medio }\end{array}$ & $\begin{array}{c}\text { 95\% Intervalo de Confianza } \\
\text { de la Media }\end{array}$ & Mín. & Máx. \\
\hline & & & $\begin{array}{c}\text { Limite } \\
\text { Inferior }\end{array}$ & $\begin{array}{c}\text { Limite } \\
\text { Superior }\end{array}$ & & \\
\hline Calidad del servicios & 437 & 91.93 & 80.0 & 90.50 & 93.35 & 45.00 & 125.00 \\
\hline
\end{tabular}




\section{Conclusiones}

El objetivo de este trabajo descriptivo fue probar y validar un instrumento para la medición de la calidad del servicio de la biblioteca de una universidad pública; en términos generales los resultados revelan el instrumento final es una herramienta con buenas propiedades psicométricas, es decir, valido y confiable para evaluar la calidad del servicio de la biblioteca en el contexto estudiado. Esto se sustenta en lo que en los siguientes párrafos se comenta.

La validez de contenido del instrumento a través del Método Lawshe modificado por Tristán muestra que en su conjunto los ítems son característicos del dominio teórico (calidad del servicio) al alcanzar un Índice de validez global (CVI) de 0.81

Con respecto a la validez de constructo, la medida de adecuación muestral KMO (0.933) y la prueba de Bartlett $\left(C^{2}=6312.655 ; p=.000\right)$ fueron sumamente pertinentes para la aplicación del análisis factorial, el cual demostró que se mantuvo la solución de cuatro variables que explican el $63.038 \%$ de la varianza total. La configuración de este instrumento quedó formada por cuatro variables, a saber: Compromiso personal (7 ítems), Biblioteca como espacio (7 ítems), Servicios de información proporcionados (6 ítems) y TIC aplicadas al servicio (5 ítems), concordando en cuanto a variables con LibQUAL+ pero no en cuanto a ítems ya que a diferencia del cuestionario original, este instrumento perdió dos ítems de los 27 que tenía en un comienzo, quedando con 25.

La confiabilidad determinada por el alfa de Cronbach en el instrumento de 25 ítems presentó un índice global de 0.929 considerándose un puntaje aceptable y bueno en los primeros estudios de validación de un instrumento, según Nunnally y Bernstein (1995). Los índices de cada una de las variables reflejaron altos índices de consistencia interna, pues oscilaron entre 0.934 y 0.778 , más altos que los reportados por Huamán, et al. (2008) para una versión castellana abreviada del cuestionario LibQUAL+ aplicado en bibliotecas de universidades peruanas.

A través de este proceso de validación fue posible advertir algunas diferencias en cuanto a la procedencia del usuario, de ahí que se recomienda que futuras investigaciones profundicen en este aspecto lo cual permitirá orientar a los responsables del servicio respecto a la elaboración de planes de mejora específicos para cada una de las bibliotecas.

Sin embargo, aun cuando la aplicación del instrumento traerá beneficios no debe olvidarse que esta investigación se realizó empleado una muestra por conveniencia por tanto los resultados deberán tomarse con reserva porque no existe la seguridad de que cada unidad muestral integre a la población total en el proceso de selección de la muestra, de ahí que no es factible hacer inferencias sobre la calidad del servicio. Esta característica se convierte en una limitante, de ahí que sería recomendable ampliar el diseño muestral para lograr una representatividad más importante. 


\section{Referencias}

Águila, V. (2005). El concepto de calidad en la educación universitaria: Clave para el logro de la competitividad institucional, Revista Iberoamericana de Educación, Vol. 35/5, en: http://www.rieoei.org/deloslectores Calidad de la Educacion.htm

Carretero-Dios, H. y Pérez, C. (2005). Normas para el desarrollo y revisión de estudios instrumentales, International Journal of Clinical and Health Psychology, Vol. 5 No. 3, pp. 521-551

Heredia, L., Sánchez, A. y Vargas, D. (2012). Confiabilidad y validez del cuestionario de espiritualidad de Parsian y Dunning en versión española. Rev. Latino-Am. Enfermagem, Vol. 20, No. 3 [acceso: agosto 2012];[8 pantallas].

Herrera-Viedma, E., López-Gijón, J., \& Ávila, B. Satisfacción de usuarios y calidad: El modelo LibQUAL y su aplicación en bibliotecas españolas e iberoamericanas, 2008. In Coloquio ALCl

Huamán, R., Alfaro, K. y Vílchez, V. (2008). Evaluación de la calidad del servicio de una biblioteca universitaria: La experiencia del LibQUAL+ en cinco facultades de la Universidad Nacional Mayor de San Marcos, Biblios, No. 3131

ISO 11620 (1998). Information and documentation - Library performance indicators. Ginebra: ISO.

Játiva, M.V. y Gallo, J.P. (2006). LibQUAL+: Evaluación de calidad de servicios desde la perspectiva del usuario, Boletín de la Anabad, Vol. 56, No. 1, pp. 53-74. http://www.anabad.org/organizacion/murcia/ATT00026.pdf

Játiva, M.V. (2012). Diseño de servicios por tipología de usuarios, Universidad de Alicante, Consultado en: http://ssyf.ua.es/va/formacion/documentos/cursos-programados/2012/promocions/promocio-a1/maria-victoria-jativa/tema-serviciostipologia-usuarios.pdf

Lawshe CH. (1975). A Quantitative approach to content Validity Personnel, Psychology, Vol. 28, pp. 563-575

Mejía, J. (2000). El muestreo en la investigación cualitativa, Investigaciones Sociales, Año IV, No. 5, consultado en: http://sisbib.unmsm.edu.pe/bibvirtualdata/publicaciones/inv sociales/n5 2000/a08.pdf

Morales, P. (2011). El análisis factorial en la construcción e interpretación de test, escalas y cuestionarios, Universidad Pontificia Comillas, Madrid, Documento disponible en: http//:www.upcomillas.es/personal/peter/investigacion/AnalisisFactorial.pdf

Nunnally JC y Bernstein I. Teoría psicométrica. 3ª ed., México: McGraw-Hill; 1995, pp. 209-28

Parasuraman, A., Zeithaml, V. y Berry I. (1985). A conceptual model of service quality and its implications for future research, Journal of Marketing, Vol. 49, 41-50.

Parasuraman, A., Zeithaml, V. y Berry, L. (1988): "SERVQUAL: A Multiple-It Scale for Measuring Customer Perceptions of Service Quality", Journal of Retailing, pp. 12-40.

Tristán, A. (2008). Modificación al modelo de Lawshe para el dictamen cuantitativo de la validez de contenido de un instrumento objetivo, Avances en medición, Vol. 6, Núm. 1, pp.37-48

Zeithaml, V.A.; Parasuraman, A.; Berry, L.L. (1990). Delivering quality service: Balancing customer perceptions and expectations. New York: Free Press. 


\section{Anexo: Composición del Instrumento de Medición}

\begin{tabular}{|c|c|}
\hline VARIABLES & ÍTEMS \\
\hline \multirow{7}{*}{$\begin{array}{l}\text { Compromiso del } \\
\text { personal }\end{array}$} & 1. El personal de la biblioteca le inspira confianza \\
\hline & 2. El personal le ofrece atención personalizada \\
\hline & 3. El personal es amable en la atención hacia usted \\
\hline & 4. El personal muestra disposición para ayudarlo \\
\hline & $\begin{array}{l}\text { 5. Considera que el personal posee amplios conocimientos para responder a sus } \\
\text { preguntas }\end{array}$ \\
\hline & 6. El personal comprende sus necesidades \\
\hline & 7. El personal brinda servicios con prontitud \\
\hline \multirow{7}{*}{$\begin{array}{l}\text { Biblioteca como } \\
\text { espacio }\end{array}$} & 20. La infraestructura de la biblioteca ayuda al estudio y el aprendizaje \\
\hline & 21. La biblioteca cuenta con espacios cómodos \\
\hline & 22. La biblioteca ofrece un ambiente tranquilo para el estudio individual \\
\hline & 23. La biblioteca otorga un ambiente favorable para el estudio y trabajo en grupo \\
\hline & 24. La biblioteca cuenta con una adecuada iluminación \\
\hline & 25. La biblioteca cuenta con una adecuada ventilación \\
\hline & 26. La biblioteca es un lugar ideal para estudiar y aprender \\
\hline \multirow{6}{*}{$\begin{array}{l}\text { Servicios de } \\
\text { información } \\
\text { proporcionados }\end{array}$} & $\begin{array}{l}\text { 8. Los libros impresos existentes en la biblioteca cubren las necesidades de } \\
\text { información que necesito }\end{array}$ \\
\hline & $\begin{array}{l}\text { 9. La cantidad de copias de los libros impresos son suficientes para mis } \\
\text { necesidades de información }\end{array}$ \\
\hline & $\begin{array}{l}\text { 10. Los libros electrónicos existentes en la biblioteca cubren las necesidades de } \\
\text { información que necesito }\end{array}$ \\
\hline & 11. Las revistas de la biblioteca cubren las necesidades de información \\
\hline & $\begin{array}{l}\text { 12. Las bases de datos electrónicas de la biblioteca cubren las necesidades de } \\
\text { información que necesito }\end{array}$ \\
\hline & $\begin{array}{l}\text { 13. La colección de recursos informativos (libros impresos, libros electrónicos, } \\
\text { dvd, enciclopedias, diccionarios etc..) disponibles en la biblioteca corresponden } \\
\text { a ediciones actualizadas }\end{array}$ \\
\hline \multirow{5}{*}{$\begin{array}{l}\text { TIC aplicadas al } \\
\text { servicio }\end{array}$} & $\begin{array}{l}\text { 15. El sitio WEB de la biblioteca http://biblioteca.uabc.mx (herramienta para } \\
\text { recuperación de información como catalogo cimarrón, bases de datos, libros } \\
\text { electrónicos) es fácil de usar y permite encontrar por mi mismo lo que busco. }\end{array}$ \\
\hline & $\begin{array}{l}\text { 16. Puedo accesar a recursos electrónicos (bases de datos, libros electrónicos) desde } \\
\text { mi hogar o lugar de trabajo }\end{array}$ \\
\hline & $\begin{array}{l}\text { 17. La biblioteca me permite el acceso a textos completos de revistas usando las } \\
\text { bases de datos electrónicas }\end{array}$ \\
\hline & $\begin{array}{l}\text { 18. La biblioteca cuenta con computadoras modernas que me permiten el acceso } \\
\text { fácil a la información que necesito }\end{array}$ \\
\hline & $\begin{array}{l}\text { 19. Los cursos de orientación para usuarios, sobre el uso de servicios y recursos de } \\
\text { información de la biblioteca, me permiten utilizar los servicios de biblioteca de } \\
\text { forma más eficiente }\end{array}$ \\
\hline
\end{tabular}

En esta tabla se muestra la composición final del instrumento atendiendo al resultado del Analisis Factorial 


\section{Datos de las autoras}

María del Carmen Toledo Sánchez

Licenciada en informática. Jefa de la Biblioteca de la Universidad Autonoma de Baja California, Campus Ensenada.

ctoledo@uabc.edu.mx

Sonia Elizabeth Maldonado-Radillo

Profesora e investigadora de la Facultad de Ciencias Administrativas y Sociales de la Universidad Autonoma de Baja California, Campus Ensenada.

se.maldonado@gmail.com

Virginia Guadalupe López Torres

Profesora e investigadora de la Facultad de Ciencias Administrativas y Sociales de la Universidad Autonoma de Baja California, Unidad Valle Dorado.

vglopeztorres@gmail.com

Recibido-Received : 2012-12-12

Aceptado-Accepted : :2012-12-30

\section{(cc) EY New articles in this journal are licensed under a Creative Commons Attribution 3.0} United States License.

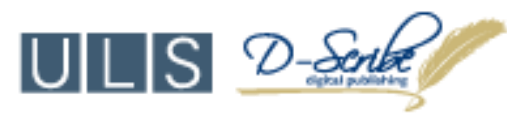

This journal is published by the University Library System of the University of Pittsburgh as part of its D-Scribe Digital Publishing Program and is cosponsored by the University of Pittsburgh Press. 